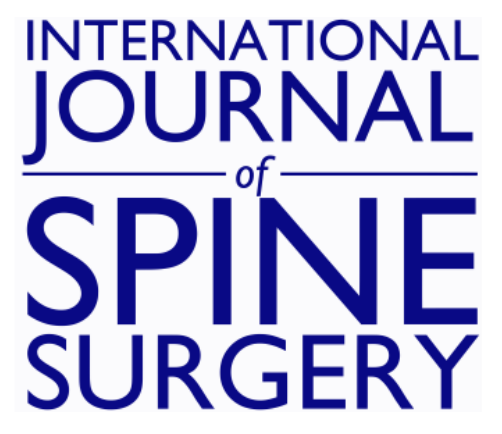

\title{
Prospective, Randomized, Multicenter FDA IDE Study of CHARITÉ Artificial Disc versus Lumbar Fusion: Effect at 5-year Follow-up of Prior Surgery and Prior Discectomy on Clinical Outcomes Following Lumbar Arthroplasty
}

Fred H. Geisler, Paul C. McAfee, Robert J. Banco, Scott L. Blumenthal, Richard D. Guyer, Richard T. Holt and Mohamed E. Majd

Int J Spine Surg 2009, 3 (1) 17-25

doi: https://doi.org/10.1016/SASJ-2008-0019-RR

http://ijssurgery.com/content/3/1/17

This information is current as of April 26, 2023.

Email Alerts Receive free email-alerts when new articles cite this article. Sign up at: http://ijssurgery.com/alerts 


\title{
Prospective, Randomized, Multicenter FDA IDE Study of CHARITÉ Artificial Disc versus Lumbar Fusion: Effect at 5-year Follow-up of Prior Surgery and Prior Discectomy on Clinical Outcomes Following Lumbar Arthroplasty
}

\author{
Fred H. Geisler, MD, PhD, ${ }^{a}$ Paul C. McAfee, MD, ${ }^{b}$ Robert J. Banco, MD, ${ }^{c}$ Scott L. Blumenthal, MD, ${ }^{d}$ \\ Richard D. Guyer, MD, ${ }^{d}$ Richard T. Holt, MD, ${ }^{e}$ Mohamed E. Majd, MD
}

\begin{abstract}
Background

Candidates for spinal arthrodesis or arthroplasty often present with a history of prior surgery such as laminectomy, laminotomy or discectomy. In this study, lumbar arthroplasty patients with prior surgery, and in particular patients with prior discectomy, were evaluated for their clinical outcomes at the 5 -year time point.
\end{abstract}

\section{Methods}

Randomized patients from the 5-year CHARITÉ investigational device exemption (IDE) study were divided as follows: 1) fusion prior surgery (excluding prior decompression with fusion) group (FSG); 2) fusion prior discectomy group (FDG); 3) fusion no prior surgery group (FNG); 4) arthroplasty prior surgery group (ASG); 5) arthroplasty prior discectomy group (ADG); and 6) arthroplasty no prior surgery group (ANG). The 5-year clinical outcomes included visual analog scale (VAS), Oswestry Disability Index 2.0 (ODI), patient satisfaction, and work status.

\section{Results}

In the arthroplasty group, all subgroups had statistically significant VAS improvements from baseline (VAS change from baseline: ASG $=-36.6 \pm 29.6, P<0.0001 ;$ ADG $=-40.2 \pm 30.9, P=0.0002 ;$ ANG $=-36.5 \pm 34.6, P<0.0001)$. There was no statistical difference between subgroups $(P=0.5587)$. In the fusion group, VAS changes from baseline were statistically significant for the FNG and FSG subgroups, but not for the FDG patients (FNG $=-46.3 \pm 28.8, P<0.0001$; FSG $=-24.2 \pm 36.4, P=0.0444$; FDG $=-26.7 \pm 38.7, P=0.2188)$. A trend of decreased VAS improvements was observed for FSG versus FNG $(P=0.0703)$ subgroups. Similar findings and trends were observed in ODI scores (Changes in ODI from baseline: ASG $=-20.4 \pm 23.8$, $P<0.0001 ; \mathrm{ANG}=-26.6 \pm 21.1, P<0.0001 ; \mathrm{ADG}=-17.6 \pm 28.6, P=0.0116 ; \mathrm{FSG}=-14.5 \pm 21.2, P=0.0303$; $\mathrm{FNG}=-32.5 \pm 22.6, P<0.0001 ; \mathrm{FDG}=-10.7 \pm 9.4, P=0.0938)$. The greatest improvement in work status from preoperative to postoperative was seen in the ADG subgroup ( $28 \%$ increase in part- and full-time employment), while the FDG subgroup showed the greatest reduction in work status ( $17 \%$ decrease).

\section{Conclusions}

Arthroplasty patients with prior surgery or prior discectomy had similar clinical outcomes as arthroplasty patients without prior surgery, while fusion patients with prior surgery or prior discectomy showed trends of lowered clinical outcomes compared to fusion patients without prior surgery or discectomy.

Key Words: arthrodesis; arthroplasty; lumbar; clinical trial; 5-year follow-up; prior surgery. SAS Journal. March 2009;3:16-24. DOI: SASJ-2008-0019-RR

${ }^{a}$ Illinois Neuro-Spine Center, 2020 Ogden Avenue, Suite 335, Aurora, IL 60504 b Spine and Scoliosis Center, Towson, MD 21204 'Boston Spine Group, Boston, MA $02120^{\text {d} T e x a s ~ B a c k ~ I n s t i t u t e, ~ P l a n o, ~ T X ~} 75093$ ' Spine Surgery, Louisville, KY 40202

Address correspondence to Dr. Fred H. Geisler, Illinois Neuro-Spine Center, 2020 Ogden Avenue, Suite 335, Aurora, IL 60504 (e-mail: fgeisler@gmail.com)

Fred Geisler, PhD, MD, consults for DePuy Spine, Inc., Aesculap, Inc., Abbott Spine, K2M, and Spinal Motion; receives royalties from DePuy and Aesculap; and owns stock in Disc Motion and Impliant, Inc. Paul McAfee, MD, owns stock in, consults for, and has received research support from DePuy Spine, Inc., Cervitech, Inc., and Globus Medical, Inc. Robert Banco, MD, is a consultant for DePuy Spine, Inc., Stryker Corporation, Medtronic, Inc., and Impliant, Inc. Scott Blumenthal, MD, is a consultant to DePuy Spine, Inc. Richard Guyer, $\mathrm{MD}$, is a consultant to and receives royalties from DePuy Spine, Inc. Mohamed E. Majd, MD is a member of the faculty with Stryker Corporation, Alphatec Spine, Inc., and DePuy Spine, Inc.

IRB approval was obtained at each of the participating study sites.

\section{INTRODUCTION}

The development of new spinal arthroplasty devices has prompted multiple level I randomized controlled trials to evaluate the clinical impact of arthroplasty versus fusion in controlled patient populations. ${ }^{1-4}$ While these studies are creating a wealth of information on the safety and effectiveness of various devices for the treatment of degenerative disc disease (DDD), their indication 
is usually restricted to a very narrowly defined patient population and, as such, they provide only limited information on the critical issue of patient selection for either fusion or arthroplasty.

A prior CHARITÉ (DePuy Spine, Raynham, Massachusetts) investigational device exemption (IDE) study, which was designed to evaluate the Artificial Disc versus BAK (Zimmer Spine, Minneapolis, Minnesota) interbody fusion with iliac crest autograft for the treatment of degenerative disc disease at 1 level from L4 to S1, also included strict inclusion and exclusion criteria that ensured a homogeneous patient population. ${ }^{1,3}$ However, the study design allowed inclusion of patients with prior laminectomies, foraminotomies or discectomies. At the 2-year time point, all the patients were analyzed and it was found that those who had undergone a prior surgery experienced similar benefits from their spinal surgery as those who had not had a prior surgery. ${ }^{5}$ No information exists, however, on the long-term benefits of fusion and arthroplasty on this specific (prior surgery) patient population.

The long-term clinical benefits of spinal fusion have been discussed in multiple reports. ${ }^{6,7}$ Long-term arthroplasty results have also been the subject of several publications ${ }^{8,9}$; however, the information included in these reports represents level IV data as none of the studies were based on multicenter, randomized controlled cases. Recently, the 5-year results from the artificial disc versus interbody fusion study were compiled, providing long-term efficacy data - for both fusion and arthroplasty-from a multicenter, randomized controlled trial. Our study of the 5 -year results provides a unique opportunity to understand the long-term impact of both fusion and arthroplasty on specific patient populations, such as patients with prior surgery as well as patients with prior discectomy.

In this study, both the arthroplasty and fusion patient populations were subdivided based on the patients' history of prior surgery or prior discectomy. The prior surgery patient subgroups were compared to the subgroups without prior surgery or discectomy.

\section{MATERIALS AND METHODS}

Study Design and Surgical Technique

Between May 2000 and April 2002, 375 patients were randomized for treatment by either anterior lumbar fusion with the interbody fusion system and iliac crest autograft or total disc replacement with the artificial disc as part of a prospective, randomized, non-blinded, FDAapproved IDE study conducted at 14 investigational sites across the United States. At the completion of the 2-year study, a new investigation was initiated to further collect data from this study, up to the 5-year time point. All 14 sites were invited to participate; however, 6 sites declined continuation, reducing the number of available patients by 90 . A total of 160 patients presented for their 5-year follow-up: 43 interbody fusion patients, 90 randomized arthroplasty cases, and 27 non-randomized (training) arthroplasty cases. Randomized cases only are included in this analysis. Patients were subdivided by prior surgery history as shown in Table 1. Prior surgery was not an exclusion criterion for the IDE study, as long as it was defined as prior decompressions via discectomy or laminotomy/foraminotomy without fusion. Prior decompression with fusion, on the other hand, was listed as an exclusion criterion. Patients in this study, therefore, do not include cases with prior fusion surgery. Of the 90 arthroplasty patients, 37 had prior surgery of which 21 had prior discectomy. Of the 43 fusion patients, 12 had prior surgery of which 6 had prior discectomy. The groups are defined as arthroplasty prior surgery group (ASG) and fusion prior surgery (excluding prior fusion) group (FSG); arthroplasty prior discectomy group (ADG) and fusion prior discectomy group (FDG); and arthroplasty no prior surgery group (ANG) and fusion no prior surgery group (FNG).

\section{Clinical Outcome Measurements}

Comparisons of clinical outcomes between patients with prior surgery, prior discectomy or no prior surgery were performed using VAS (0-100) and ODI scores preoperatively, at 6 weeks, and at 3-, 6-, 12-, 24- and 60 -months postoperative. At 12-, 24- and 60-months postoperative, additional analyses were conducted to compare patient satisfaction and return to work status between groups.

\section{Statistical Methods}

Data were analyzed using the SAS v8.2 statistical software package (SAS Institute, Cary, North Carolina). For categorical variables, $\mathrm{P}$ values were generated using Fisher's exact test. A t test was used to test means.

\section{RESULTS}

\section{Demographics}

Demographicinformation was compiled and compared for all groups, as shown in Table 1. No statistical difference was observed between groups. In the arthroplasty group, a majority of females had prior surgery or prior discectomy. This trend was not observed in the fusion group. Average age, height, weight, and BMI were also not statistically different between groups. A majority of patients were treated at L5-S1 in all groups except the FDG, where the same number of procedures was performed at both L4-L5 and L5-S1.

\section{Surgical Data}

Surgical times, blood loss and hospitalization days are shown in Table 2. There were no statistical differences for these variables between the prior surgery/prior 


\begin{tabular}{|c|c|c|c|c|c|c|}
\hline \multirow[b]{2}{*}{ Demographics } & \multicolumn{3}{|c|}{ Arthroplasty } & \multicolumn{3}{|c|}{ Fusion } \\
\hline & Prior Surgery & Discectomy & Vo Prior Surgery & Prior Surgery & Discectomy & No Prior Surgery \\
\hline Number of Subjects & 37 & 21 & 53 & 12 & 6 & 31 \\
\hline \multicolumn{7}{|l|}{ Gender } \\
\hline Female & $14(38 \%)$ & $9(43 \%)$ & $29(55 \%)$ & $6(50 \%)$ & $3(50 \%)$ & $13(42 \%)$ \\
\hline Male & $23(62 \%)$ & $12(57 \%)$ & $24(45 \%)$ & $6(50 \%)$ & $3(50 \%)$ & $18(58 \%)$ \\
\hline \multicolumn{7}{|l|}{ Age (Years) } \\
\hline Mean (Std) & $38.4(8.48)$ & $40.1(8.64)$ & $41.0(8.57)$ & $35.3(5.89)$ & $37.8(5.56)$ & $40.2(9.28)$ \\
\hline Median & 40 & 43 & 41 & 36 & 38.5 & 42 \\
\hline Min, Max & 19,55 & 20,55 & 21,60 & 25,43 & 28,43 & 25,55 \\
\hline \multicolumn{7}{|l|}{ Height (cm) } \\
\hline Mean (Std) & $175.4(8.72)$ & $174.5(9.09)$ & $172.5(9.86)$ & $172.5(9.71)$ & $173.1(10.46)$ & $173.8(8.77)$ \\
\hline Median & 175.3 & 175.3 & 172.7 & 174 & 174 & 172.7 \\
\hline Min, Max & 157,193 & 157,191 & 150,191 & 155,185 & 155,185 & 157,191 \\
\hline \multicolumn{7}{|l|}{ Weight (kg) } \\
\hline Mean (Std) & $80.8(17.73)$ & 79.3 (17.59) & $79.4(14.97)$ & $83.1(14.04)$ & $80.8(19.31)$ & $81.0(16.71)$ \\
\hline Median & 81.6 & 81.2 & 80.7 & 86.2 & 86 & 77.1 \\
\hline Min, Max & 51,120 & 51,120 & 52,111 & 54,109 & 54,109 & 54,118 \\
\hline \multicolumn{7}{|l|}{ Body Mass Index } \\
\hline Mean (Std) & $26.1(4.45)$ & $25.9(4.71)$ & $26.6(3.92)$ & $28.1(5.13)$ & $27.0(6.31)$ & $26.7(4.69)$ \\
\hline Median & 26.6 & 27.1 & 26.3 & 28.6 & 27.3 & 26.3 \\
\hline Min, Max & 19,34 & 19,33 & 17,37 & 19,36 & 19,36 & 19,40 \\
\hline \multicolumn{7}{|l|}{ Targeted Level } \\
\hline L4-5 & $10(27 \%)$ & $4(19 \%)$ & $16(30 \%)$ & $5(42 \%)$ & $3(50 \%)$ & $5(16 \%)$ \\
\hline L5-S1 & $27(73 \%)$ & $17(81 \%)$ & $37(70 \%)$ & $7(58 \%)$ & $3(50 \%)$ & $26(84 \%)$ \\
\hline & \multicolumn{3}{|c|}{ Arthroplasty } & \multicolumn{3}{|c|}{ Fusion } \\
\hline Demographics & Prior Surgery & Discectomy & No Prior Surgery & Prior Surgery & Discectomy & No Prior Surgery \\
\hline $\mathrm{N}$ & 37 & 21 & 53 & 12 & 6 & 31 \\
\hline \multicolumn{7}{|c|}{ Total Surgery Time (Min) } \\
\hline Mean (Std) & $97.5(29.44)$ & $95.6(29.42)$ & $115.6(53.69)$ & $106.3(66.58)$ & $81.8(29.14)$ & $128.2(66.23)$ \\
\hline Median & 88 & 87 & 99 & 74 & 67.5 & 115 \\
\hline Min, Max & 56,175 & 56,175 & 45,250 & 55,248 & 62,137 & 60,355 \\
\hline \multicolumn{7}{|c|}{ Estimated Blood Loss (cc) } \\
\hline Mean (Std) & $158.3(143.3)$ & $145.2(124.65)$ & $248.7(277.81)$ & $251.6(440.72)$ & $341.5(618.46)$ & $186.0(169.19)$ \\
\hline Median & 100 & 100 & 150 & 75 & 99.5 & 150 \\
\hline Min, Max & 50,600 & 50,500 & 25,1500 & 50,1600 & 50,1600 & 20,700 \\
\hline \multicolumn{7}{|c|}{ Duration of Hospital Stay } \\
\hline Mean (Std) & $3.7(0.97)$ & $3.8(1.22)$ & $3.7(.86)$ & $4.3(2.50)$ & $3.5(0.55)$ & $4.3(1.47)$ \\
\hline Median & 4 & 4 & 4 & 4 & 3.5 & 4 \\
\hline Min, Max & 1,6 & 1,6 & 2,6 & 3,12 & 3,4 & 2,8 \\
\hline
\end{tabular}

discectomy subgroups and the no prior surgery patients in both the fusion and arthroplasty groups. In the arthroplasty group, patients with prior surgery or discectomy showed trends of reduced surgical time and blood loss compared to patients with no history of prior surgery. No difference in hospitalization days was found between the 3 arthroplasty subgroups. In the fusion group, a similar trend of reduced surgical time was observed between prior surgery/prior discectomy patients and those patients without prior surgery. However, this trend was reversed 
Figure 1.

A Changes in VAS Scores for Arthroplasty Cases

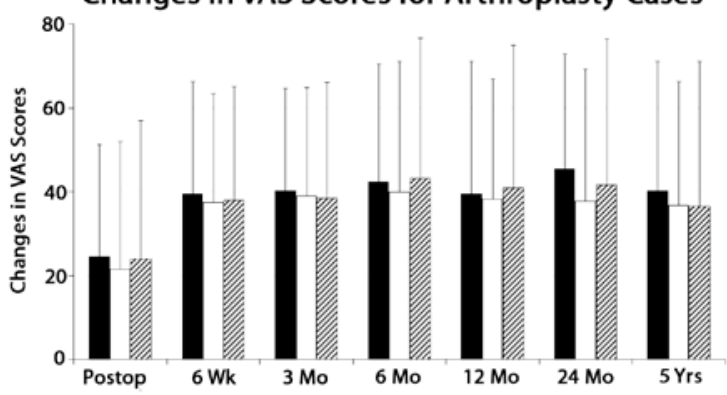

B

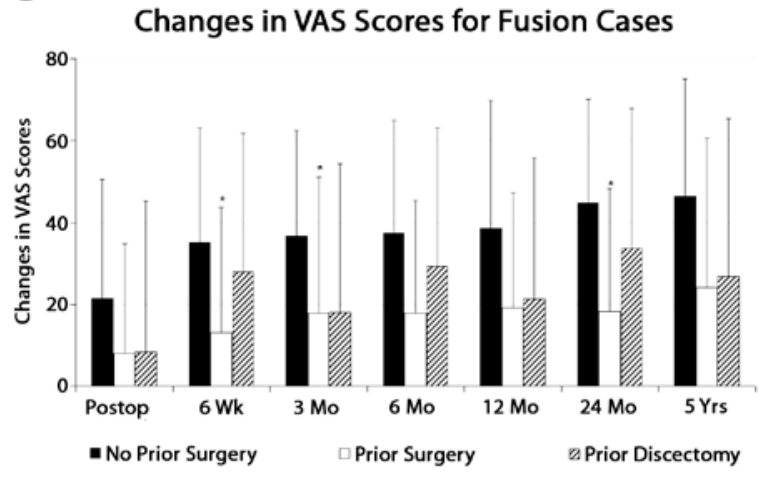

Changes in VAS scores for arthroplasty (A) and fusion (B) patients, by time point. The "**" indicates statistical significance compared to the no prior surgery subgroups (ANG or FNG).

with regard to blood loss. There were no differences in hospitalization days among all 3 fusion subgroups.

\section{VAS and ODI}

VAS scores for both arthroplasty and fusion patients are shown in Figures 1A and 1B. In the arthroplasty group, all subgroups showed similar changes in VAS at all time points. In all arthroplasty subgroups, changes from baseline to 5 years were statistically significant (change from baseline: $\mathrm{ASG}=-36.6 \pm 29.6, P<0.0001 ; \mathrm{ADG}=-40.2 \pm 30.9$, $P=0.0002 ; \mathrm{ANG}=-36.5 \pm 34.6, P<0.0001)$. In addition, the difference in VAS changes from baseline between the ASG and the ANG subgroups was not statistically significant $(P=0.5587)$. In the fusion group, changes from baseline were also statistically significant for the FNG subgroup (VAS change from baseline: $\mathrm{FNG}=-46.3 \pm 28.8, P<0.0001)$. However, the VAS improvements from baseline were not statistically significant in the FDG subgroup, and they were barely significant in the FSG subgroup (change from baseline: $\mathrm{FSG}=-24.2 \pm 36.4$, $P=0.0444 ; \mathrm{FDG}=-26.7 \pm 38.7, P=0.2188)$. In addition, the difference in VAS changes from baseline between the FSG and FNG subgroups showed a trend of decreased VAS improvements for FSG $(P=0.0703)$.

VAS improvements were also compared between ANG and FNG, ASG and FSG, as well as ADG and FDG. Despite the fairly large differences in mean scores

\section{Figure 2.}

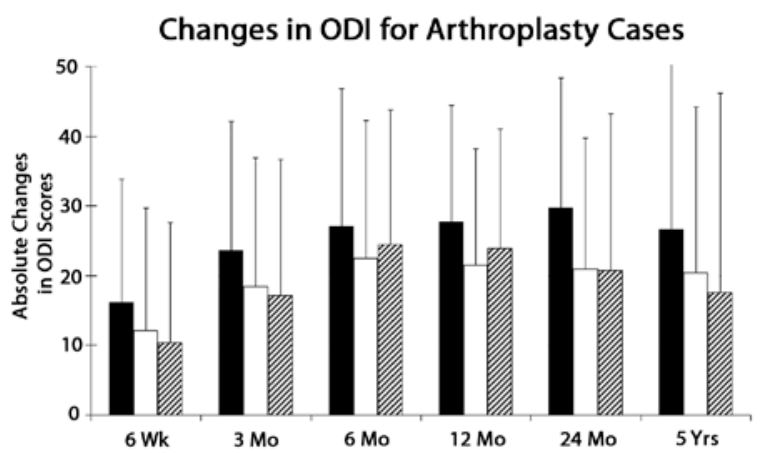

Changes in ODI for Fusion Cases

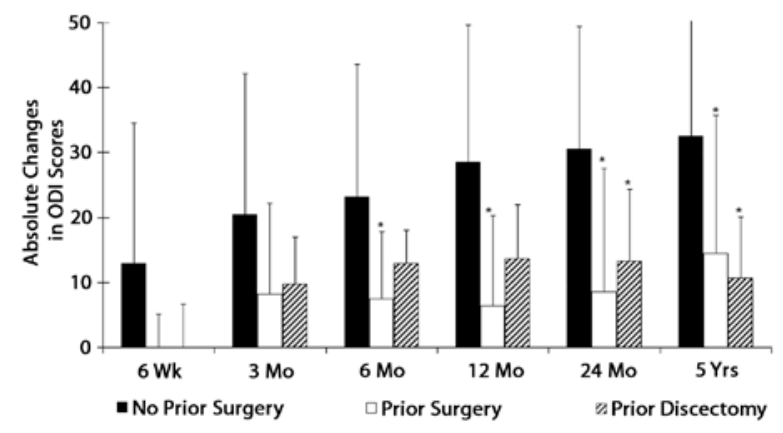

Changes in ODI scores for arthroplasty (A) and fusion (B) patients, by time point. The $"$ "** indicates statistical significance compared to the no prior surgery subgroups (ANG or FNG)

between the arthroplasty and fusion subgroups, none were statistically significant.

ODI scores for both arthroplasty and fusion patients are shown in Figures $2 \mathrm{~A}$ and $2 \mathrm{~B}$. In the arthroplasty group, all subgroups showed statistical improvements from baseline to 5 years. In addition, there was no statistical difference in ODI improvements between the ANG and ASG subgroups $(P=0.2245)$, and between the ANG and ADG subgroups $(P=0.1406)$. (Changes in ODI from baseline: $\mathrm{ASG}=-20.4 \pm 23.8, P<0.0001$; $\mathrm{ANG}=-26.6 \pm 21.1, P<0.0001 ; \mathrm{ADG}=-17.6 \pm 28.6$, $P=0.0116)$. In the fusion group, changes from baseline were statistically significant for the FNG subgroup (Changes in ODI: $\mathrm{FNG}=-32.5 \pm 22.6, P<0.0001$ ); however, improvements in ODI were not statistically significant in the FDG subgroup and were barely significant for the FSG subgroup $(\mathrm{FDG}=-10.7 \pm 9.4$, $P=0.0938 ; \mathrm{FSG}=-14.5 \pm 21.2, P=0.0303)$. In addition, there was a statistically significant difference between ODI improvements for the FNG subgroup versus the FSG $(P=0.0356)$, and for the FNG versus the FDG $(P=0.0273)$ subgroup.

ODI improvements were also compared between the subgroups $\mathrm{ANG}$ and $\mathrm{FNG}, \mathrm{ASG}$ and $\mathrm{FSG}$, as well as ADG and FDG. Again, despite fairly large differences in mean scores, none were statistically significant. 


\section{Return to Work}

The return to work status by treatment group and time point is shown in Figure 3. The greatest improvement in work status from preoperative to postoperative was seen in the ADG subgroup: Improvement in work status reached $7 \%, 25 \%$ and $28 \%$ in the $\mathrm{ANG}, \mathrm{ASG}$ and $\mathrm{ADG}$ subgroups, respectively. In the fusion group, improvement in work status from preoperative to 5-year postoperative was observed only in the FSG subgroup (42\% to $50 \%$ ); it declined in the FNG (62\% to 61\%) and FDG subgroups $(67 \%$ to $50 \%)$.

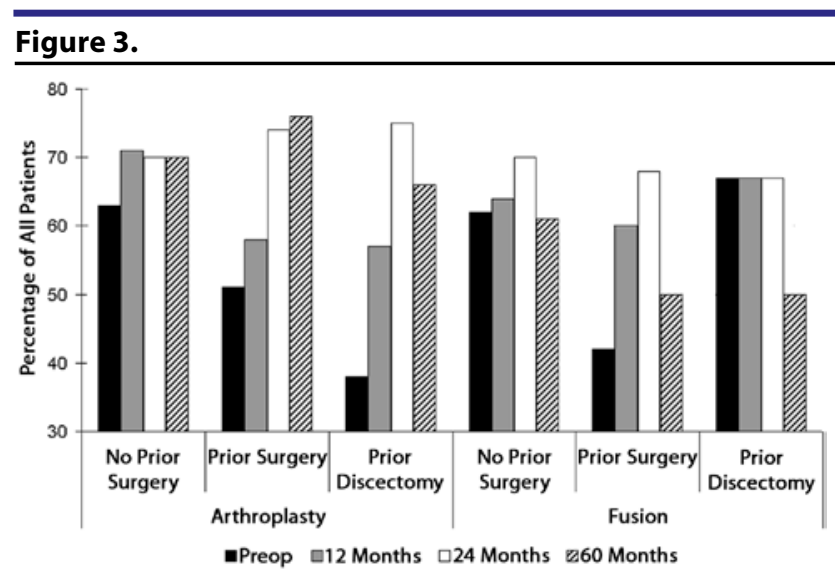

Percentage of patients employed in full- or part-time jobs, by subgroups and time points.

At the 5-year postoperative time point, there were $26 \%$ more ASG patients with full- or part-time work versus FSG patients, and there were $16 \%$ more patients with full- or part-time work in the ADG subgroup versus the FDG. In the no prior surgery subgroups, there was only a $9 \%$ difference between arthroplasty and fusion subjects in terms of work status.

\section{Patient Satisfaction}

Patient satisfaction with the procedure at 12-, 24- and 60months is shown in Figure 4. At the 5-year time point, patient satisfaction ranged from $81 \%$ (ADG) to $97 \%$ (ANG). There were no statistical differences in patient satisfaction at that time point.

\section{DISCUSSION}

The purpose of this study was to analyze the clinical outcomes of arthroplasty and fusion patients with and without prior surgery or prior discectomy. Only randomized cases were included herein. Overall, arthroplasty patients with prior surgery or prior discectomy experienced clinical outcomes that were similar to those experienced by arthroplasty patients without prior surgery or prior discectomy. However, fusion patients who had undergone prior surgery or prior discectomy experienced smaller pain and disability improvements (as measured by VAS and ODI) compared to fusion patients without prior surgery or prior discectomy.

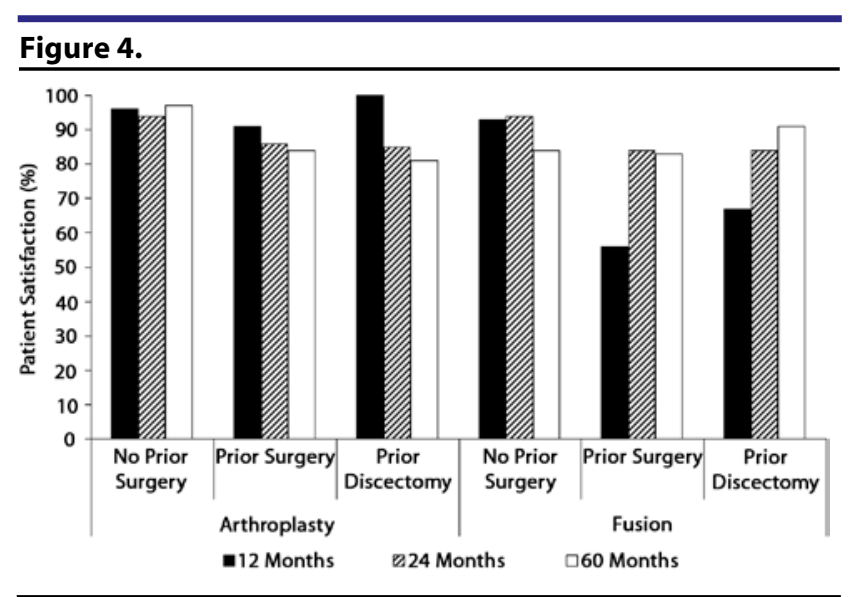

Percentage of patients who were "satisfied" or "somewhat satisfied" with the procedure at the various time points and by subgroups.

In all subgroups, average differences in outcomes at the 5-year time point exceeded the minimum clinically important differences (MCID) established for VAS $(18-19 \mathrm{~mm})$ and ODI (10 points). ${ }^{10}$ In the arthroplasty group, VAS improvements were twice the MCID for all subgroups, while ODI improvements were twice the MCID for the ANG and ASG subgroups but not the ADG. In the fusion group, VAS and ODI improvements were twice the MCID for the FNG but not for the FSG and FDG subgroups, in which VAS and ODI improvements barely reached the MCID threshold.

The return to work percentage was also greater in the arthroplasty group than in the fusion group. This finding was already apparent in the overall IDE 5-year followup, ${ }^{11}$ which showed a statistically greater percentage of arthroplasty patients returning to work versus fusion patients. In our current analyses, while statistical significances were not shown, fusion patients with prior discectomy and prior surgery experienced the lowest rates of return to work. In comparison, arthroplasty patients with prior surgery had the greatest rate of return to work.

Despite the small sample size in the fusion prior surgery and fusion prior discectomy group, these results may further question whether such procedures (eg, decompressions with laminotomy/foraminotomy or discectomy) should be considered contraindications for subsequent fusion procedures with BAK and iliac crest autograft.

Limitations of this study include the unequal distribution of patients across the groups analyzed, the loss of patients to follow-up and the subsequent small sample sizes at the 5-year time point. It is possible that among the loss to follow-up patients are patients who have brought legal claims for personal injuries rather than continuing in the study. Due to lack of information and other constraints, we are not presently able to determine 
the significance of these patients' outcomes to the study. This is in part due to the fact that the IDE study was initially designed for a 2-year follow-up. Thus, while all of the 14 sites involved in the initial 2-year IDE trial were invited to participate in the 5-year follow-up, 6 declined continuation. Since all sites initially signed up for a 2year study, they were under no obligation to pursue the investigation beyond the 2 -year time point. The resulting small patient samples, especially in the prior discectomy subgroups, prevented in-depth statistical analyses.

\section{CONCLUSION}

Arthroplasty patients with or without prior surgery or prior discectomy experienced, on average, significant clinical improvements from their surgery. All subgroups exceeded MCID values for mean VAS and ODI improvements, and in most cases, they showed a clinical improvement equal to twice the MCID values.

Similarly, all fusion patients met, on average, the MCID values for VAS and ODI. However, the prior surgery and prior discectomy subgroups showed trends of reduced clinical outcomes as compared to fusion patients without prior surgery or discectomy. Fusion patients with prior discectomy and prior surgery had the lowest rate of return to work.

This article was submitted on August 11, 2008, and accepted for publication on February 23, 2009.

The authors thank Brian Hetzell and George DeMuth from Stat-Tech Services for statistical analyses, and Dr. Chantal Holy, Director of Scientific Affairs for DePuy Spine, Inc., for editorial support.

\section{REFERENCES}

1. Blumenthal S, McAfee PC, Guyer RD, et al. A prospective, randomized, multicenterFood and DrugAdministration investigational device exemptions study of lumbar total disc replacement with the CHARITÉ Artificial Disc versus lumbar fusion: part I: evaluation of clinical outcomes. Spine. 2005;30:1565-1575.

2. Le Huec JC, Mathews H, Basso Y, et al. Clinical results of Maverick lumbar total disc replacement: two-year prospective follow-up. Orthop Clin North Am. 2005;36:315-322.

3. McAfee PC, Cunningham B, Holsapple G, et al. A prospective, randomized, multicenter Food and DrugAdministration investigational device exemption study of lumbar total disc replacement with the CHARITÉ Artificial Disc versus lumbar fusion: part II: evaluation of radiographic outcomes and correlation of surgical technique accuracy with clinical outcomes. Spine. 2005;30:1576-1583.

4. Zigler J, Delamarter R, Spivak JM, et al. Results of the prospective, randomized, multicenter Food and Drug Administration investigational device exemption study of the ProDisc-L total disc replacement versus circumferential fusion for the treatment of 1-level degenerative disc disease. Spine. 2007;32:1155-1162.

5. Geisler FH, Guyer RD, Blumenthal SL, et al. Effect of previous surgery on clinical outcome following 1-level lumbar arthroplasty. J Neurosurg Spine. 2008;8:108-114.
6. Brantigan JW, Neidre A, Toohey JS. The Lumbar I/F Cage for posterior lumbar interbody fusion with the variable screw placement system: 10-year results of a Food and Drug Administration clinical trial. Spine J. 2004;4:681-688.

7. Kuslich SD, Danielson G, Dowdle JD, et al. Four-year follow-up results of lumbar spine arthrodesis using the Bagby and Kuslich lumbar fusion cage. Spine. 2000;25:2656-2662.

8. Lemaire JP, Carrier H, SariAli E, Skalli W, Lavaste F. Clinical and radiological outcomes with the CHARITÉ Artificial Disc: a 10-year minimum follow-up. J Spinal Disord Tech. 2005;18:353-359.

9. David T. Long-term results of one-level lumbar arthroplasty: minimum 10-year follow-up of the CHARITÉ Artificial Disc in 106 patients. Spine. 2007;32:661-666.

10. Hagg O, Fritzell P, Nordwall A, Swedish Lumbar Spine Study Group. The clinical importance of changes in outcome scores after treatment for chronic low back pain. Eur Spine J. 2003;12:12-20.

11. Guyer RD, McAfee P, Banco RJ, et al. Prospective, randomized, multicenter Food and Drug Administration investigational device exemption study of lumbar total disc replacement with the CHARITÉ Artificial Disc versus lumbar fusion: 5-year follow-up. Proceedings of the CNS/AANS Joint Section. 2008. 


\section{EXTENDED REFERENCES}

A prospective, randomized, multicenter Food and Drug Administration investigational device exemptions study of lumbar total disc replacement with the CHARITE artificial disc versus lumbar fusion: part I: evaluation of clinical outcomes.

Blumenthal S, McAfee PC, Guyer RD, Hochschuler SH, Geisler FH, Holt $R T$, Garcia R Jr, Regan JJ, Ohnmeiss DD.

STUDY DESIGN: A prospective, randomized, multicenter, Food and Drug Administration-regulated Investigational Device Exemption clinical trial. OBJECTIVES: The purpose of this study was to compare the safety and effectiveness of lumbar total disc replacement, using the CHARITE artificial disc (DePuy Spine, Raynham, MA), with anterior lumbar interbody fusion, for the treatment of single-level degenerative disc disease from L4-S1 unresponsive to nonoperative treatment. SUMMARY OF BACKGROUND DATA: Reported results of lumbar total disc replacement have been favorable, but studies have been limited to retrospective case series and/or small sample sizes. METHODS: Three hundred four (304) patients were enrolled in the study at 14 centers across the United States and randomized in a 2:1 ratio to treatment with the CHARITE artificial disc or the control group, instrumented anterior lumbar interbody fusion. Data were collected pre- and perioperatively at 6 weeks and at 3, 6, 12, and 24 months following surgery. The key clinical outcome measures were a Visual Analog Scale assessing back pain, the Oswestry Disability Index questionnaire, and the SF-36 Health Survey. RESULTS: Patients in both groups improved significantly following surgery. Patients in the CHARITE artificial disc group recovered faster than patients in the control group. Patients in the CHARITE artificial disc group had lower levels of disability at every time interval from 6 weeks to 24 months, compared with the control group, with statistically lower pain and disability scores at all but the 24 month follow-up $(P<0.05)$. At the 24-month follow-up period, a significantly greater percentage of patients in the CHARITE artificial disc group expressed satisfaction with their treatment and would have the same treatment again, compared with the fusion group $(P<0.05)$. The hospital stay was significantly shorter in the CHARITE artificial disc group $(P<0.05)$. The complication rate was similar between both groups. CONCLUSIONS: This prospective, randomized, multicenter study demonstrated that quantitative clinical outcome measures following lumbar total disc replacement with the CHARITE artificial disc are at least equivalent to clinical outcomes with anterior lumbar interbody fusion. These results support earlier reports in the literature that total disc replacement with the CHARITE artificial disc is a safe and effective alternative to fusion for the surgical treatment of symptomatic disc degeneration in properly indicated patients. The CHARITE artificial disc group demonstrated statistically significant superiority in two major economic areas, a 1-day shorter hospitalization, and a lower rate of reoperations (5.4\% compared with $9.1 \%$ ). At 24 months, the investigational group had a significantly higher rate of satisfaction $(73.7 \%)$ than the $53.1 \%$ rate of satisfaction in the control group $(P=0.0011)$. This prospective randomized multicenter study also demonstrated an increase in employment of $9.1 \%$ in the investigational group and $7.2 \%$ in the control group.

A prospective, randomized, multicenter Food and Drug Administration investigational device exemption study of lumbar total disc replacement with the CHARITE artificial disc versus lumbar fusion: part II: evaluation of radiographic outcomes and correlation of surgical technique accuracy with clinical outcomes.

McAfee PC, Cunningham B, Holsapple G, Adams K, Blumenthal S, Guyer $R D$, Dmietriev A, Maxwell JH, Regan JJ, Isaza J.

STUDY DESIGN: A prospective, randomized, multicenter, Food and Drug Administration-regulated, investigational device exemption clinical trial. OBJECTIVES: To compare the safety and effectiveness of lumbar total disc replacement (TDR) with the CHARITE artificial disc (DePuy Spine, Raynham, MA) to anterior lumbar interbody fusion for the treatment of single-level degenerative disc disease from L4-S1 unresponsive to nonoperative treatment. In addition, to evaluate the radiographic outcomes of lumbar artificial disc replacement at either L4-L5 or L5-S1 with the CHARITE artificial disc as compared to anterior lumbar interbody fusion with cylindrical cages and iliac crest bone graft; and to determine if a correlation exists between clinical outcomes and surgical accuracy of TDR placement within the disc space. SUMMARY OF BACKGROUND DATA: Prior investigators have reported excellent radiographic results with the CHARITE artificial disc for the treatment of lumbar degenerative disc disease. These encouraging results are the product of retrospective reviews without a control. Very few studies have reported on the segmental motion of an intervertebral level implanted with an artificial disc, and no studies have reported a correlation of radiographic and clinical outcomes. METHODS: A prospective, randomized, multicenter, US Food and Drug Administration, investigational device exemption study with 24-month follow-up was performed at 14 centers throughout the United States. A total of 304 subjects were randomized in a 2:1 ratio, with 205 in the investigational group (TDR with the CHARITE artificial disc) and 99 in the control group (anterior lumbar interbody fusion with BAK cages and iliac crest bone graft). A total of 71 TDR training cases were performed (up to 5 at each site) before randomization beginning at each site. Plain radiographs were analyzed for each subject in both groups regarding range of motion (ROM) in flexion/extension, restoration of disc space height, and subsidence. Prosthesis placement in the coronal and midsagittal planes was analyzed for the 276 patients with TDR. Correlations were performed between prosthesis placement and clinical outcomes. RESULTS: Patients in the investigational group had a $13.6 \%$ mean increase, and those in the control group an $82.5 \%$ decrease in mean flexion/extension ROM at 24 months postoperatively compared to baseline. Patients in the investigational group had significantly better restoration of disc height than the control group $(P<0.05)$. There was significantly less subsidence in the investigational group compared to the control group $(P<0.05)$. The surgical technical accuracy of CHARITE artificial disc placement was divided into 3 groups: I, ideal (83\%); II, suboptimal (11\%); and III, poor $(6 \%)$, and correlated with clinical outcomes. The flexion/extension ROM and prosthesis function improved with the surgical technical accuracy of radiographic placement $(P=0.003)$. CONCLUSIONS: Preoperative ROM in flexion/extension was restored and maintained in patients receiving a TDR. TDR with the CHARITE artificial disc resulted in significantly better restoration of disc space height, and significantly less subsidence than anterior interbody fusion with BAK cages. Clinical outcomes and flexion/extension ROM correlated with surgical technical accuracy of CHARITE artificial disc placement. In the majority of cases, placement of the CHARITE artificial disc was ideal.

Effect of previous surgery on clinical outcome following 1-level lumbar arthroplasty.

Geisler FH, Guyer RD, Blumenthal SL, McAfee PC, Cappuccino A, Bitan F, Regan JJ.

OBJECT: A secondary lumbar surgery at a previously surgically treated level is believed to result in minimal clinical improvement. The clinical results of the CHARITE Investigational Device Exemption (IDE) study were analyzed to assess the effect of previous surgery on clinical outcomes following either total disc replacement with the CHARITE device or anterior lumbar interbody fusion with a BAK cage and iliac crest autograft. METHODS: Patients with prior microdiscectomy, laminectomy, or minimal medial facetectomy were not excluded from enrollment in the CHARITE IDE study. Thus, the following 3 groups were analyzed: all patients treated with the CHARITE Artificial Disc, whether randomized or nonrandomized; only patients treated with CHARITE devices randomized against patients with BAK devices; and control patients with BAK devices. Each group was further subdivided based on the patients' medical history, whether they had undergone prior surgery (prior surgery group) or had not (no prior surgery group). For all groups, baseline demographics were collected and compared for any 
potential recruitment bias. Postoperative improvements based on Oswestry Disability Index (ODI), visual analog scale (VAS), and patient satisfaction scores were further collected and statistically analyzed. RESULTS: For all 3 groups, there were no statistical differences in clinical improvement from 3 months to 2 years postoperatively as measured using ODI and VAS scores between the subgroups (those who had prior surgery and those who did not). CONCLUSIONS: Patients indicated for 1-level lumbar arthroplasty with previous lumbar decompressive surgery can be expected to have similar clinical outcomes to patients undergoing arthroplasty without prior lumbar decompressive surgery. Similarly, candidates for anterior lumbar fusion with prior decompressive surgery may experience similar benefits from the surgical procedure as those without.

The Lumbar I/F Cage for posterior lumbar interbody fusion with the variable screw placement system: 10-year results of a Food and Drug Administration clinical trial.

Brantigan JW, Neidre A, Toohey JS.

BACKGROUND CONTEXT: The Lumbar I/F Cage is a carbon fiber reinforced polymer (CFRP) device designed to separate the mechanical and device functions of interbody fusion. A Investigational Device Exemption (IDE) clinical study of the CFRP cage was conducted during an enrollment period from 1991 to 1993. Based on the 2-year results of this study, the cage was approved by the US Food and Drug Administration (FDA) in February 1999. Since then, the Lumbar I/F Cage device has become widely used in the United States. PURPOSE: This study was designed to determine the long-term results of patients who received this device during the 1991-1993 enrollments. STUDY DESIGN/SETTING: Investigators from the original study were asked to evaluate their original patients according to FDAreviewed case report forms. Although many of the centers were unable to provide significant follow-up, two centers that enrolled almost half of the original study group provided reports on a high percentage of their original patients. This paper reviews the results in those patients. PATIENT SAMPLE: Inclusion criteria included patients with degenerative disc disease who had at least one failed lumbar discectomy or decompression procedure at one or more levels. OUTCOME MEASURES: Clinical success was defined by a modified Prolo score evaluating pain, function, medication usage and economic status. Fusion success, determined by evaluation of plain radiographs, was defined by continuous bone bridging the fusion area with no lucencies. Flexion-extension X-rays were done on patients who had previous removal of pedicle screw implants. Any motion on flexion-extension films indicated pseudarthrosis. METHODS: All patients were contacted at their last known address. Internet search services were used to locate additional patients. Thirty-three of 43 eligible patients $(77 \%)$ were evaluated, including 31 patients who reported for examination and X-ray and 2 additional patients by telephone survey and written questionnaire. RESULTS: Clinical success was achieved in 32 of 37 patients $(86.5 \%)$ at 24 months and in 29 of 33 patients $(87.8 \%)$ at 10 years. This included $61 \%$ excellent, $27 \%$ good, and $12 \%$ fair results. Fusion success was reported in 37 of 37 patients (100\%) at 24 months and in 29 of 30 patients $(96.7 \%)$ at 10 years. Patient satisfaction was reported in 31 of $33(93.9 \%)$. Further lumbar surgery was done in 23 patients: in 18 patients for elective removal of pedicle screws and in 5 patients to extend the fusion to adjacent levels. Adjacent segment degeneration occurred in $61 \%$ of patients but was clinically significant in only $20 \%$. Smokers had equal clinical and fusion success with nonsmokers at 24 months and 10 years and had adjacent segment degeneration in $37 \%$, a rate significantly lower than nonsmokers at $87 \%$. CONCLUSIONS: The high rate of clinical success, fusion success, and patient satisfaction at 24 months was maintained at 10 -year follow-up. Adjacent segment degeneration was common but was usually not clinically significant.

Long-term results of one-level lumbar arthroplasty: minimum 10-year follow-up of the CHARITE artificial disc in 106 patients.
STUDY DESIGN: A retrospective chart and radiographic review. OBJECTIVES: To determine the long-term clinical results, radiographic results, and incidence of complications in a large patient cohort with one-level lumbar total disc replacement (TDR). SUMMARY OF BACKGROUND DATA: Prior authors have described short-term, midterm, and long-term clinical and radiographic results in patients with lumbar TDR with highly variable results. METHODS: From January 1989 to November 1995,108 patients, with a mean age of 36.4 years, underwent lumbar TDR with the CHARITE Artificial Disc, with 106 available for follow-up. A modified Stauffer-Coventry scale was used to determine clinical outcome. Return to work, work level, and the incidence of complications were assessed. Dynamic lateral flexion-extension and lateral bending radiographs were performed and segmental range of motion (ROM) was measured using the Cobb method. RESULTS: Mean follow-up time was 13.2 years (range, 10-16.8 years). Of the 106 patients, $87(82.1 \%)$ had either an excellent or good clinical outcome. Of the 96 patients working before surgery, 86 returned to work (89.6\%), including $77.8 \%$ of patients with hard labor level employment ( 28 of 36 ) returning to the same level of work. The mean ROM in flexion-extension was 10.1 degrees , in lateral bending it was 4.4 degrees , and $90.6 \%$ of implanted prostheses were still mobile. Eight patients $(7.5 \%)$ required posterior instrumented fusion. There were 5 cases $(4.6 \%)$ of postoperative facet arthrosis, 3 cases $(2.8 \%)$ of subsidence, 3 cases $(2.8 \%)$ of adjacent-level disease, and 2 cases $(1.9 \%)$ of core subluxation. CONCLUSIONS: This retrospective study demonstrates the safety and efficacy of the CHARITE Artificial Disc at one level, either L4-L5 or L5-S1, in the long-term. Clinical outcomes and the rate of return to work were excellent overall. The rate of adjacent-level disease requiring surgical intervention was considerably lower $(2.8 \%)$ compared with reports in the literature for lumbar fusion. As with any surgical procedure, proper indications play a pivotal role in clinical success. 


\section{SUPPLEMENTARY DATA}

Table 1. Posterior Lumbar Fusions in the United States by Gender, 2004-2007

\begin{tabular}{lcc}
\hline By Gender & Count & $\%$ \\
$\%$ Male & 8,733 & $43.2 \%$ \\
$\%$ Female & 11,471 & $56.8 \%$ \\
Total & 20,204 & $100.0 \%$ \\
\hline
\end{tabular}

Table 2. Average Charges for Posterior Lumbar Fusion in the United States by Gender, 2004-2007

\begin{tabular}{lcc}
\hline By Gender & Count & Average Charge \\
Male & 8733 & $\$ 79,795$ \\
Female & 11471 & $\$ 79,903$ \\
\hline \multicolumn{3}{l}{} \\
\hline \multicolumn{3}{l}{$\begin{array}{l}\text { Table 3. 360 degree } \\
\text { Gender, 2004-2007 }\end{array}$} \\
\hline By Gender & Count & $\%$ \\
$\%$ Male & 1,121 & $41.9 \%$ \\
$\%$ Female & 1,552 & $58.1 \%$ \\
Total & 2,673 & $100.0 \%$ \\
\hline
\end{tabular}

Table 4. Average Charge for 360 degree Lumbar Fusions in the United States by Gender, 2004-2007

\begin{tabular}{lcc}
\hline By Gender & Count & Average Charge \\
$\%$ Male & 1,121 & $\$ 126,777$ \\
$\%$ Female & 1,552 & $\$ 125,696$ \\
\hline \multicolumn{2}{c}{ Table 5. Average Charges for Posterior Lumbar Fusions in the } \\
\hline United States by Region, 2007 & \\
\hline Region & Average Charge \\
Northeast & $\$ 79,785$ \\
Midwest & $\$ 79,401$ \\
West & $\$ 109,985$ \\
South & $\$ 93,169$ \\
\hline
\end{tabular}

Table 6. Average Charges for Posterior Lumbar Fusions in the United States by Year

\begin{tabular}{ccc}
\hline Year & Average Charge & \% Change \\
2004 & $\$ 68,397$ & \\
2005 & $\$ 73,527$ & $7.5 \%$ \\
2006 & $\$ 81,728$ & $11.2 \%$ \\
2007 & $\$ 91,275$ & $11.7 \%$ \\
\hline
\end{tabular}

Table 7. Average Charges for 360 degree Lumbar Fusions in the United States by Year

\begin{tabular}{ccc}
\hline Year & Average Charge & \% Change \\
2004 & $\$ 109,079$ & \\
2005 & $\$ 112,151$ & $2.8 \%$ \\
2006 & $\$ 133,203$ & $18.8 \%$ \\
2007 & $\$ 141,056$ & $5.9 \%$ \\
\hline
\end{tabular}

PearlDiver data regarding the clinical procedures/diagnoses described in this paper is derived from HIPAA compliant insurance, private payer sources. These tables and charts are for information purposes only. The PearlDiver data has been obtained or derived from sources believed by
Table 8. Postoperative Complications by ICD-9 Code, Posterior Lumbar Fusion in the United States, 2004-2007

\begin{tabular}{|c|c|c|}
\hline & Count & $\%$ \\
\hline Total Posterior Lumbar Fusions & 20,204 & \\
\hline \multicolumn{3}{|l|}{ Cardiac } \\
\hline $997.1,410.0-410.9,998.0$ & 148 & $0.73 \%$ \\
\hline \multicolumn{3}{|l|}{ Respiratory } \\
\hline $997.3,415.1,518.0-518.4$ & 965 & $4.78 \%$ \\
\hline \multicolumn{3}{|l|}{ Peripheral } \\
\hline 997.2 & 7 & $0.03 \%$ \\
\hline \multicolumn{3}{|l|}{ CNS } \\
\hline $997.0,997.00,997.01,997.09$ & 291 & $1.44 \%$ \\
\hline \multicolumn{3}{|l|}{ Hematoma } \\
\hline $998.1,998.11,998.12,998.13$ & 691 & $3.42 \%$ \\
\hline \multicolumn{3}{|l|}{ Accidental cut / hemorrage } \\
\hline $998.2, \mathrm{E} 870.0$ & 646 & $3.20 \%$ \\
\hline \multicolumn{3}{|l|}{ Complication of operative wound } \\
\hline $998.3,998.31,998.32,998.83$ & 125 & $0.62 \%$ \\
\hline \multicolumn{3}{|l|}{ Postoperative infection } \\
\hline $998.5,999.3,998.51,998.59$ & 446 & $2.21 \%$ \\
\hline \multicolumn{3}{|l|}{ Other unspecified complications } \\
\hline $\begin{array}{l}\text { 998.8, 998.89,998.9,999.9, } \\
\text { E8788,E8789 }\end{array}$ & 434 & $2.15 \%$ \\
\hline \multicolumn{3}{|l|}{ CSF leak } \\
\hline $349.0,998.6$ & 57 & $0.28 \%$ \\
\hline \multicolumn{3}{|l|}{ Vertebral injury } \\
\hline $\begin{array}{l}900.00,900.01,900.02,900.03 \\
900.82,900.89,900.9,997.02,954.0\end{array}$ & 3 & $0.01 \%$ \\
\hline \multicolumn{3}{|c|}{ Mechanical Compliation of Implant or Graft } \\
\hline $996.4,996.40$ & 888 & $4.40 \%$ \\
\hline
\end{tabular}

Table 9. Postoperative Complications, 360 degree Lumbar Fusion in the United States, 2004-2007

\begin{tabular}{|c|c|c|}
\hline & Count & $\%$ \\
\hline Total 360 Lumbar Fusions & 2,673 & \\
\hline \multicolumn{3}{|l|}{ Cardiac } \\
\hline $997.1,410.0-410.9,998.0$ & 27 & $1.01 \%$ \\
\hline \multicolumn{3}{|l|}{ Respiratory* } \\
\hline $997.3,415.1,518.0-518.4$ & 178 & $6.66 \%$ \\
\hline \multicolumn{3}{|l|}{ Peripheral } \\
\hline 997.2 & 1 & $0.04 \%$ \\
\hline \multicolumn{3}{|l|}{ CNS } \\
\hline $997.0,997.00,997.01,997.09$ & 30 & $1.12 \%$ \\
\hline \multicolumn{3}{|l|}{ Hematoma } \\
\hline $998.1,998.11,998.12,998.13$ & 106 & $3.97 \%$ \\
\hline \multicolumn{3}{|l|}{ Accidental cut / hemorrage } \\
\hline 998.2, E870.0 & 108 & $4.04 \%$ \\
\hline \multicolumn{3}{|l|}{ Complication of operative wound } \\
\hline $998.3,998.31,998.32,998.83$ & 20 & $0.75 \%$ \\
\hline \multicolumn{3}{|l|}{ Postoperative infection } \\
\hline $998.5,999.3,998.51,998.59$ & 48 & $1.80 \%$ \\
\hline \multicolumn{3}{|l|}{ Other unspecified complications } \\
\hline $\begin{array}{l}\text { 998.8, 998.89,998.9,999.9, } \\
\text { E8788,E8789 }\end{array}$ & 71 & $2.66 \%$ \\
\hline \multicolumn{3}{|l|}{ CSF leak } \\
\hline $349.0,998.6$ & 1 & $0.04 \%$ \\
\hline \multicolumn{3}{|l|}{ Vertebral injury } \\
\hline $\begin{array}{l}900.00,900.01,900.02,900.03,900.82,90 \\
0.89,900.9,997.02,954.0\end{array}$ & 1 & $0.04 \%$ \\
\hline \multicolumn{3}{|c|}{ Mechanical Complication of Implant or Graft } \\
\hline $996.4,996.40$ & 174 & $6.51 \%$ \\
\hline
\end{tabular}

PearlDiver to be reliable, but PearlDiver Technologies Inc. does not represent that the information is $100 \%$ accurate or complete. For more information, go to http://pearldiverinc.com 